\title{
Reward-driven enhancements in motor control are robust to TMS manipulation
}

\author{
Olivier Codol ${ }^{1}$ · Joseph M. Galea ${ }^{1} \cdot$ Roya Jalali $^{1}$ P Peter J. Holland ${ }^{1}$
}

Received: 15 January 2020 / Accepted: 1 April 2020 / Published online: 9 April 2020

(c) The Author(s) 2020

\begin{abstract}
A wealth of evidence describes the strong positive impact that reward has on motor control at the behavioural level. However, surprisingly little is known regarding the neural mechanisms which underpin these effects, beyond a reliance on the dopaminergic system. In recent work, we developed a task that enabled the dissociation of the selection and execution components of an upper limb reaching movement. Our results demonstrated that both selection and execution are concommitently enhanced by immediate reward availability. Here, we investigate what the neural underpinnings of each component may be. To this end, we aimed to alter the cortical excitability of the ventromedial prefrontal cortex and supplementary motor area using continuous theta-burst transcranial magnetic stimulation (cTBS) in a within-participant design $(N=23)$. Both cortical areas are involved in determining an individual's sensitivity to reward and physical effort, and we hypothesised that a change in excitability would result in the reward-driven effects on action selection and execution to be altered, respectively. To increase statistical power, participants were pre-selected based on their sensitivity to reward in the reaching task. While reward did lead to enhanced performance during the cTBS sessions and a control sham session, cTBS was ineffective in altering these effects. These results may provide evidence that other areas, such as the primary motor cortex or the premotor area, may drive the reward-based enhancements of motor performance.
\end{abstract}

keywords Motor control $\cdot$ Reaching $\cdot$ Theta-burst TMS $\cdot$ Reward $\cdot$ Action selection $\cdot$ Action execution

\section{Introduction}

In saccadic eye movements, reward has a well-known ability to invigorate motor control, enhance accuracy, and promote accurate action selection in the face of potential distractors (Kojima and Soetedjo 2017; Manohar et al. 2015; Sohn and Lee 2006; Takikawa et al. 2002). Recently, we extended these behavioural findings from eye movements to reaching movements (Codol et al. 2020). Specifically, we found that reward enhanced action selection by increasing participants' propensity to move towards the correct target in the presence of a distractor target, while reaction times were not impeded. Execution of reaching movements also showed a pronounced increase in peak velocity (vigour) with

Communicated by Sven Bestmann.

Olivier Codol

codol.olivier@gmail.com

1 School of Psychology, University of Birmingham, Birmingham, UK reward, while radial accuracy was maintained. While these reward-driven improvements are now behaviourally wellcharacterised and confirmed in a number of previous reports (Griffiths and Beierholm 2017; Reppert et al. 2018; Summerside et al. 2018), the neural substrates of these effects remain unknown. Here, we aimed to investigate which cortical regions are involved in the reward-based enhancement of motor performance using transcranial magnetic stimulation (TMS).

During a sensorimotor task, a stream of information contributes to the generation of movement, travelling from visual and proprio-tactile sensory afferents to high-level prefrontal and parietal associative areas; then forming into a motor plan in the supplementary motor area (SMA) and pre-motor cortices, to finally produce a motor command which travels from the primary motor cortex (M1) to the spinal cord and to the effector muscles (Castiello 2005; Hikosaka et al. 2002; Shadmehr and Krakauer 2008; Thorpe and Fabre-Thorpe 2001). Therefore, to pin down the neural substrates of reward-driven enhancements, one can ask at which point of this sensory-prefrontal-premotor-motor loop 
does reward influence the processing stream. We applied continuous theta-burst transcranial magnetic stimulation (cTBS; Huang et al. 2005; Zenon et al. 2015) immediately prior to participants performing an arm reaching task. Such manipulation has been shown to alter neural activity of M1 and SMA, likely through a decrease in cortical excitability, for 20 min following cessation of stimulation (M1: Huang et al. 2005; SMA: Legon et al. 2013). The targeted regions would therefore continue to be altered for the entire duration of our behavioural task, without the need to stimulate during task performance.

Some evidence for potential cTBS targets for influencing action selection comes from the literature on attentional processes. For example, imaging studies show that occipital regions exhibit the most sensitivity to reward in attentional tasks in humans (Anderson 2016; Tosoni et al. 2013), suggesting that reward-driven selection improvements may be due to early enhancement of visual sensory processing. Alternatively, the posterior and anterior cingulate cortices, and ventro-medial prefrontal cortex (vmPFC) are also involved in reward processing (Blair et al. 2013; Daw et al. 2005, 2006; Graybiel 2008; Klein-Flugge et al. 2016), and are heavily dependent on dopamine innervation (Arnsten 1998). There is also evidence showing that vmPFC encodes the value of different stimuli during a decision-making task involving motor effort (Klein-Flugge et al. 2016). Consequently, occipital or prefrontal areas could both be considered as potential targets for cTBS. However, since occipital areas are not only involved in reward processing but also a large array of core visual functions, cTBS in these regions could potentially alter basic motor performance, and thus expose any results to unnecessary confounds. Therefore, we focus on prefrontal cTBS manipulations in this study to assess action selection susceptibility to reward. While the anterior cingulate cortex and the vmPFC are both possible candidates, the anterior cingulate cortex cannot be stimulated using cTBS due to its deep location. We therefore tested our hypothesis by targeting the vmPFC.

Regarding execution, reward-based improvements may be similarly due to enhanced encoding of visual information, thereby allowing more vigorous movements at no accuracy cost. However, this would not explain previous reports of reward-driven increase in feedback control (Carroll et al. 2019; Manohar et al. 2019) and end-point stiffness (Codol et al. 2020) during reaching tasks. Rather, reward could directly modulate M1, as M1 activity has been shown to be highly sensitive to reward (Bundt et al. 2016; Galaro et al. 2019; Kapogiannis et al. 2008; Mawase et al. 2016, 2017; Ramkumar et al. 2016; Thabit et al. 2011; Zhao et al. 2018), shaping processing near the end of the sensorimotor arc. Another reasonable hypothesis is that reward information is integrated earlier on, with M1 being merely the final recipient. Several prefrontal regions upstream of M1 are involved in action planning, including the SMA, a region also showing strong sensitivity to reward (Hendrix et al. 2018; Klein-Flugge et al. 2016; Mazzoni et al. 2007; Rascol et al. 1994; Stanford et al. 2013; Zenon et al. 2015). While cTBS stimulation over M1 would not answer whether reward is integrated in M1 or earlier on, SMA stimulation could provide more conclusive evidence. If an effect on rewarddriven enhancement of execution performance is seen, this would confirm that reward information is indeed integrated earlier than might be initially expected for reaching movements (Mawase et al. 2016, 2017; Thabit et al. 2011).

Consequently, the aim of this study was first to assess whether previously reported findings regarding the effect of reward on this reaching task replicate across multiple days within pre-selected participants who initially exhibit these beneficial effects; and second, to alter the effect of reward on action selection and action execution through cTBS of the vmPFC and SMA, respectively. Importantly, while cTBS of the $\mathrm{vmPFC}$ has been reported to be effective, the duration of the effect following cessation of stimulation is still unknown. Our study therefore assumes that the impact of cTBS is similarly sustained in the vmPFC as reported for M1 and SMA (Huang et al. 2005; Legon et al. 2013).

\section{Methods}

\section{Participants}

26 of 34 screened participants (see "screening session" section for details) were selected based on their performance on the reaching task. Of those 26 selected participants, one was later excluded due to medical reasons, and two participants retracted after the second session. Therefore, 23 participants (median age: 22, range: $18-39,15$ female) took part in the experiment and were remunerated $£ 15 /$ hour in addition to performance-based monetary rewards during the reaching task. All participants were right-handed, free of epilepsy, familial history of epilepsy, motor, psychological or neurological conditions, or any medical condition forbidding the use of cTBS or MRI. The study was approved by and completed in accordance with the University of Birmingham Ethics Committee.

\section{Task design}

The behavioural task was identical to the first experiment of Codol et al. (2020), except that only 0p (pence) and 50p trials were used. Participants performed the tasks on an endpoint KINARM (BKIN Technologies, Ontario, Canada). They held a robotic handle that could move freely on a horizontal plane in front of them, with the handle and their hand hidden by a panel (Fig. 1a). The panel included a mirror that 
A
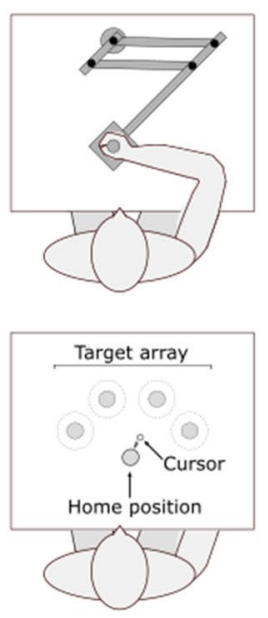

E
B

\section{C}

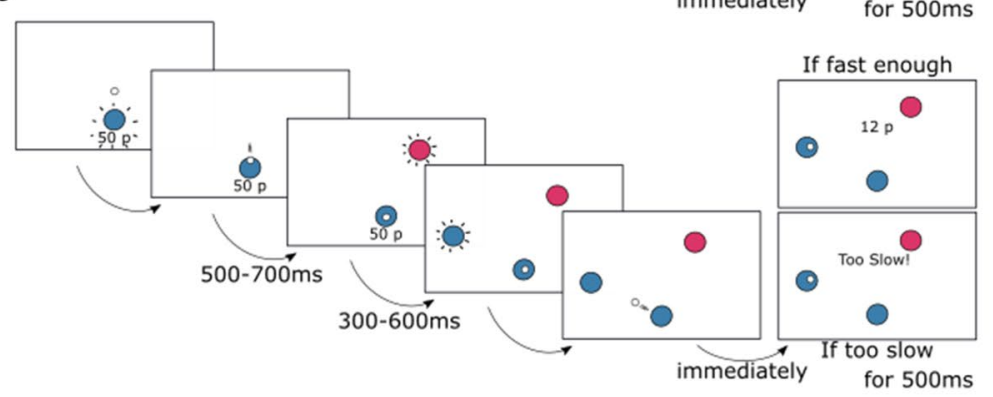

D
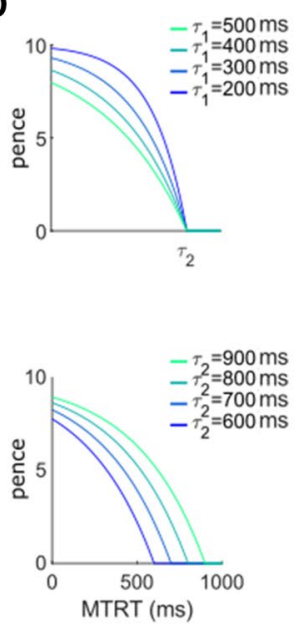

$\mathbf{F}$
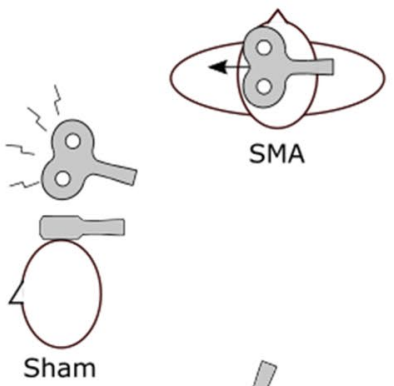

G
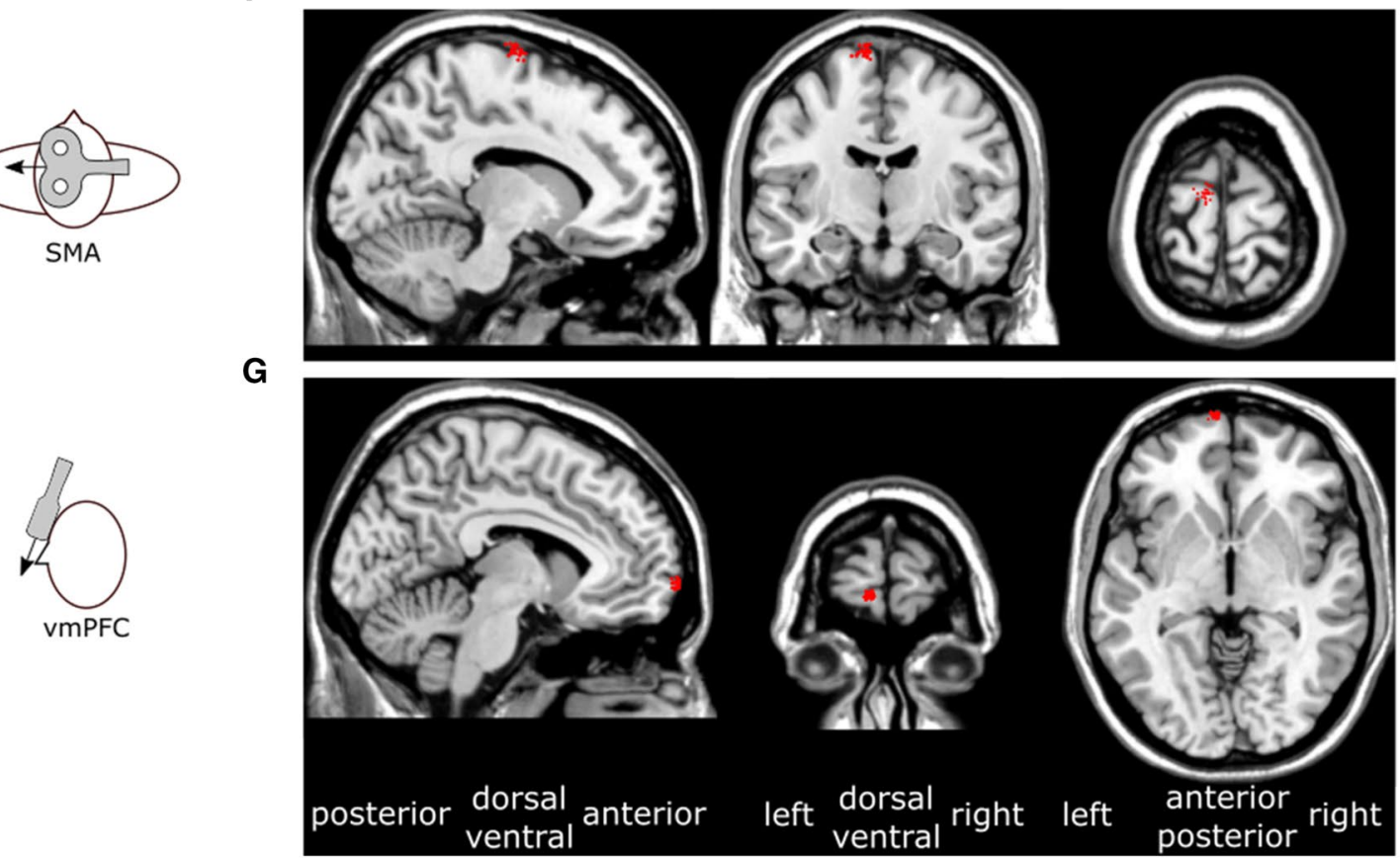

Fig. 1 Behavioural experiment and cTBS procedure. a Participants reached a series of targets using a robotic manipulandum. b Timecourse of a normal trial. Participants reached at a single target and earned money based on their performance speed (sum of movement time and reaction time; MTRT). If they were too slow (MTRT $<\tau_{2}$ ), a message "Too slow!" appeared instead of the reward information. Transition times are indicated below for each screen. A uniform distribution was employed for the transition time jitter. c Time-course of a distractor trial. Occasionally, a distractor target appeared, indicated

reflected a screen above it, and participants performed the task by looking at the reflection of the screen $(60 \mathrm{~Hz}$ refresh rate), which appeared at the level of the hidden hand. Kinematics data were sampled at $1 \mathrm{kHz}$. by a colour different from the starting position. Participants were told to wait for the second, correct target to appear and reach toward the latter. d The reward function (here for a $10 \mathrm{p}$ trial) varied based on two parameters $\tau_{1}$ (upper plot; $\tau_{2}$ fixed at $800 \mathrm{~ms}$ ) and $\tau_{2}$ (lower plot; $\tau_{1}$ fixed at $400 \mathrm{~ms}$ ). e Position of the cTBS coil(s) relative to the head in each of the 3 conditions. The black arrows represent the current orientation. f Sagittal, coronal and axial planes of an MNI-normalised brain scan (ch2.nii.gz in MRIcron). The red dots indicate each participant's SMA stimulation sites. $\mathbf{g}$ vmPFC stimulation sites

Each trial started with the robot handle bringing participants to a point $4 \mathrm{~cm}$ in front of a fixed starting position. A $2 \mathrm{~cm}$ diameter starting position (angular size $\sim 3.15^{\circ}$ ) then appeared, with its colour indicating the reward value of that trial. The reward value was also displayed in $2 \mathrm{~cm}$-high text 
(angular size $\sim 3.19^{\circ}$ ) under the starting position (Fig. 1b, c). Because colour luminance can affect salience and therefore detectability, luminance-adjusted colours were employed (see https://www.hsluv.org/) and colours assigned to distractors or real targets were counterbalanced across participants. For a given participant, the two colours coding for the real targets were never the same as the two colours coding for distractor targets.

From 500 to $700 \mathrm{~ms}$ after participants entered the starting position (on average $587 \pm 354 \mathrm{~ms}$ after the starting position appeared), a $2 \mathrm{~cm}$ diameter target (angular size $\sim 2.48^{\circ}$ ) appeared $20 \mathrm{~cm}$ away from the starting position, in the same colour as the starting position. Participants were instructed to move as fast as they could towards it and stop in it. They were informed that a combination of their reaction time and movement time defined how much money they would receive, and that this amount accumulated across the experiment. They were also informed that end-position was not factored in as long as terminated the movement within $4 \mathrm{~cm}$ of the target centre. There were 4 possible target locations positioned every $45^{\circ}$ around the midline of the workspace, resulting in a $135^{\circ}$ span (Fig. 1a).

The reward function was of a closed-loop design that incorporated the recent history of performance, to ensure that participants received similar amounts of reward despite idiosyncrasies in individuals' reaction times and movement speed. Furthermore, the closed-loop nature of the reward function ensured that the task remained consistently challenging over the course of the experiment (Berret et al. 2018; Manohar et al. 2015; Reppert et al. 2018). To that end, the reward function was defined as follows:

$r_{\mathrm{t}}=r_{\max } \cdot \max \left(1-\mathrm{e}^{\left(\frac{\mathrm{MTRT}-\tau_{2}}{\tau_{1}}\right)}, 0\right)$,

where $r_{\max }$ was the maximum reward value for a given trial, MTRT the sum of reaction time and movement time, and $\tau_{1}$ and $\tau_{2}$ adaptable parameters varying as a function of performance (Fig. 1d). Specifically, $\tau_{1}$ was the mean of the last 20 trials' 3rd and 4th fastest MTRTs, and $\tau_{2}$ was the median of the last 20 trials' 16 th and 17 th fastest MTRTs; $\tau_{1}$ and $\tau_{2}$ were initialised as 400 and $800 \mathrm{~ms}$ at the start of each participant training block, respectively. $\tau$ values were constrained so that $\tau_{1}<\tau_{2}<900$ was always true. In practice, all reward values were rounded up to the nearest penny so that only integer penny values would be displayed.

Targets were always of the same colour as the starting position (Fig. 1b), but occasional distractor targets appeared, indicated by a different colour than the starting position (Fig. 1c). Participants were informed to ignore these targets and wait for the second target to appear. Failure to comply resulted in no monetary gain for this trial. The first target (distractor or not) appeared 500-700 ms after entering the starting position using a uniform random distribution, and correct targets in distractor trials appeared 300-600 ms after the distractor target using the same distribution.

When reaching movement velocity passed below a 0.03 $\mathrm{m} / \mathrm{s}$ threshold, the end position was recorded, and monetary gains were indicated at the centre of the workspace. After $500 \mathrm{~ms}$, the robotic arm then brought the participant's hand back to the initial position $4 \mathrm{~cm}$ above the starting position.

\section{Procedure}

The experiment took place over five sessions, with a gap of at least 5 days between sessions. The first session was a screening session, in which participants were selected based on their performance during the behavioural task. In the second session, a structural MRI scan of each participant's brain was acquired, and used for the third to fifth session, during which participants performed the behavioural task after receiving either sham, SMA or vmPFC cTBS (Fig. 1a). The order of stimulation was pseudo-randomly counterbalanced across participants. Before every session, participant's health condition was assessed in accordance to the guidelines of the Ethics Committee of the University of Birmingham (UK).

\section{Screening session}

In the first session, participants were first screened for medical or psychological conditions that could exclude them from the study. They were then introduced to the cTBS technique by reading a leaflet, and they could ask any questions they wished to the experimenter. Next, they were exposed to theta-burst stimulation on their forearm to get acquainted with the sensation of stimulation. Their active motor threshold (AMT) was then determined by finding the minimal single-pulse transcranial magnetic stimulation intensity on M1 that resulted in the visible contraction of the first dorsal interossei (FDI) muscle of the preactivated right hand in 5 out of 10 trials. Finally, participants performed the behavioural task.

Participants first practiced the task in a 48-trial training block with a 25-p trial value. They were informed that money obtained during the training would not count toward the final amount they would receive. Please note that this training block was only included in the first session, to familiarise participants with the apparatus and the instructions. The starting position and target colours were all grey during training. They then performed a 16 trials, distractor-free baseline block with $0 \mathrm{p}$ and 50p trials and were informed that their score now counted toward their final monetary gain. Finally, they experienced a 224-trial main block that included 96 (42.9\%) distractor-containing trials randomly interspaced. For the three cTBS sessions, participants repeated the same task, with the exception of 
the training block which was removed. Because this study aimed to manipulate a previously characterised effect, participants were selected for the subsequent sessions only if they showed a reward driven increase in both peak velocity and selection accuracy.

Using the resulting behavioural data, participants were then screened for an effect of reward on execution and selection accuracy. Specifically, participants were expected to show an increase in peak velocity and selection accuracy (i.e. increased propensity to ignore a distractor target) in rewarded trials compared to non-rewarded trials, including one that increased by at least $10 \%$ compared to non-rewarded trials. For instance, if a participant showed an increase of $2 \%$ and $1 \%$ for peak velocity and action selection, respectively, this was considered an overall weak effect and the participant was not selected. This selection resulted in eight participants out of 34 being excluded from the study.

\section{cTBS procedure}

Using a 3-T Philips (Amsterdam, The Netherlands) scanner, high-resolution T1-weighted images were acquired for each participant $(1 \times 1 \times 1 \mathrm{~mm}$ voxel size, 175 slices in sagittal orientation). The image was then normalised to an MNI template using an affine (12-parameter) transformation (Jenkinson and Smith 2001; Jenkinson et al. 2002) with the software Statistical Parametric Mapping 12 (SPM12, London, UK). Regions of interest were then marked using MRIcron (Rorden and Brett 2000). The MNI coordinates used were $x=-8 / y=-9 / z=77$ for the SMA and $-7 / 71 /-4$ for the vmPFC (Fig. 1b, c). More specifically, the SMA target region was the posterior part of the superior frontal gyrus, or the most prominent posterior part of Brodmann area 6 (Arai et al. 2012; Zenon et al. 2015); the vmPFC target region was the most anterior part of medial orbitofrontal gyrus, or Brodmann area 10 near the limit with Brodmann 11 (Blair et al. 2013; Lev-Ran et al. 2012). These positions were all in the left hemisphere (Arai et al. 2012; Lev-Ran et al. 2012) since all our participants were right-handed. The marked scans were then transformed back into their original space using each participant's inverse transform with SPM12, and the position of each mark was manually inspected and adjusted to the closest location minimising distance between the target position and the scalp (Galea et al. 2010; Huang et al. 2005), giving subject-specific target locations. The resulting marked individual scans were then imported to a BrainSight 2 neuronavigation system (Rogue Research Inc, Montreal, Quebec), and each region of interest was targetted with the TMS coil using its motion-capture tracking function. The SMA stimulation was performed at $-90^{\circ}$ from the midline and the vmPFC stimulation was performed at $0^{\circ}$ from the midline, with the coil being placed tangencially to the forefront (i.e. almost vertically for the vmPFC, see Fig. 1e).
cTBS was applied with a figure-of-eight, $80 \mathrm{~mm}$ diameter coil (Magstim Co Ltd, Whitland, UK). We employed the continuous theta-burst stimulation technique, with one cycle lasting $40 \mathrm{~s}$, at $80 \%$ AMT or $48 \%$ intensity, whichever was the lowest. A total of 200 burst trains were applied at a frequency of $5 \mathrm{~Hz}$, with 3 pulses per burst and a pulse frequency of $50 \mathrm{~Hz}$ - giving a total amount of 600 pulses. These parameters were all based on Huang et al. (2005) and Galea et al. (2010). During all cTBS sessions (including the sham session), participants were asked if they felt fine immediately after the stimulation was performed, and upon confirmation, were asked to move approximately two meters from the stimulation chair to the chair on which they could perform the behavioural task.

\section{Data analysis}

The pre-registered a priori hypotheses, cTBS procedure, dataset and analysis scripts are all available online on the Open Science Framework website (https://osf.io/tnkrj /). Analyses were performed using custom Matlab scripts (Matworks, Natick, MA). Bayesian analyses were performed using JASP (JASP, Amsterdam, The Netherlands).

Trials were manually classified as distracted or non-distracted. Trials that did not include a distractor target-i.e. no-distractor trials-were all considered non-distracted. Distracted trials were defined as trials where a distractor target was displayed, and participants initiated their movement (i.e. exited the starting position) toward the distractor instead of the correct target. If participants readjusted their reach "midflight" to the correct target or initiated their movement to the correct target and readjusted their reach to the distractor, this was still considered a distracted trial.

Reaction times were measured as the time between the correct target onset and when the participant's distance from the centre of the starting position exceeded $2 \mathrm{~cm}$. In trials that were marked as "distracted" (i.e. participant initially went to the distractor target), the distractor target onset was used. In distractor-containing trials, the second, correct target did not require any selection process to be made, since the appearance of the distractor target informed participants that the next target would be the correct one. For this reason, reaction times were biased toward a faster range in non-distracted trials. Consequently, mean reaction times were obtained by including only no-distractor trials, and distracted trials. For every other summary variable, we included all trials that were not distracted trials, that is, we included non-distracted trials and no-distractor trials.

Trials with reaction times higher than $1000 \mathrm{~ms}$ or less than $200 \mathrm{~ms}$, and non-distracted trials with radial errors higher than $6 \mathrm{~cm}$ or angular errors higher than $20^{\circ}$ were removed. Overall, this accounted for $0.49 \%$ of all trials. Speed-accuracy functions were obtained for each participant 
by binning data in the $x$-dimension into 50 quantiles and averaging all $y$-dimension values in a $x$-dimension sliding window of a 30-centile width (Manohar et al. 2015). Then, each individual speed-accuracy function was averaged by quantile across participants in both the $x$ and $y$ dimension.

\section{Statistical analysis}

In the pre-registration of this study, we indicated that group statistics would be performed using a $2 \times 3$ repeated-measure ANOVA, with reward value ( $0 p$ versus $50 p$ ) as the first factor, and cTBS group (sham, SMA, vmPFC) as the second factor. However, because main effects were only detected in the first factor $(0 p-50 p)$ and no effect was found in the cTBS condition, we also performed post-hoc Bayesian analyses to assess the evidence in favour of the null hypothesis regarding cTBS manipulation. Results were identical regarding significant effects in the frequentist versus Bayesian approach. Frequentist ANOVAs were performed in MatLab, and Bayesian statistics were done using the Bayesian repeatedmeasure ANOVA function in JASP with mean summary statistics pre-computed and exported as csv files using MatLab. Results are reported as Bayes factors for each model against the null model $\left(\mathrm{BF}_{10}\right)$ and for each model against the best model $\left(\mathrm{BF}_{\text {best }}\right)$. A BF of 1 indicates that there is no evidence in favour of the null or the alternative model, i.e. the data is ambiguous (Wagenmakers et al. 2011). A BF that tends toward 0 indicates increasing evidence toward the null model, and inversely, a BF that tends toward $+\infty$ indicate stronger evidence for the alternative. Note that this is a logscale, i.e. a BF of 2 is as much evidence for the alternative model than a BF of 0.5 is for the null (Wagenmakers et al. 2011).

The default prior parameters were used, i.e. a Cauchy prior with $r$-scale of 0.5 for fixed effects (there was no random effect or covariate). Sampling values for numerical accuracy and model-averaged posteriors were left in the "automatic" position. To obtain model-averaged posteriors, the posterior density function of a given factor level coefficient must be averaged across all models, with each posterior density being weighted by the probability of its respective model. In other words, it is a weighted mean of posterior effects across all models. For all plotted variables, bootstrapped $95 \%$ confidence intervals of the mean were obtained using 10,000 permutations.

\section{Results}

Similar to Codol et al. (2020), reward improved both the selection and execution components of reaching movements (Fig. 1). Specifically, reward led to faster reaction times $\left(F(1,22)=8.18, p=0.009\right.$, partial $\eta^{2}=0.37$;
Fig. 1a), whilst also improving selection accuracy $\left(F(1,22)=16.7, p<0.001\right.$, partial $\eta^{2}=0.76$; Fig. $\left.1 b\right)$, clearly demonstrating that the selection component benefited from the presence of reward. Of note, the decrease of reaction times with reward in this study is surprising, as no significant effect had been observed in the same task in a previous study - though a non-significant trend in that direction could be observed (Codol et al. 2020). Regarding execution, peak velocity increased with reward $\left(F(1,22)=42.4, p<0.001\right.$, partial $\eta^{2}=1.93$; Fig. 1c $)$ whilst movement time decreased $(F(1,22)=24.0, p<0.001$, partial $\eta^{2}=1.09$; Fig. $\left.1 \mathrm{~d}\right)$. In addition, radial error $\left(F(1,22)=2.88, p=0.10\right.$, partial $\eta^{2}=0.13$; Fig. $\left.1 \mathrm{e}\right)$ and angular error $\left(F(1,22)=2.98, p=0.10\right.$, partial $\eta^{2}=0.14$; Fig. 1f) were similar across rewarded and non-rewarded trials.

In contrast, while we expected to observe an effect of cTBS on the reward-driven effects, we observed no main effect or interaction effects for cTBS: reaction times (cTBS: $F(2,44)=0.05, p=0.95$, partial $\eta^{2}=0.002$; interaction: $F(2,44)=0.65, p=0.53$, partial $\eta^{2}=0.03$; Fig. 1a), selection accuracy (main effect of cTBS: $F(2,44)=0.40, p=0.70$, partial $\eta^{2}=0.02$; interaction: $F(2,44)=1.12, p=0.33$, partial $\eta^{2}=0.05$; Fig. 1b), peak velocity (cTBS: $F(2,44)=0.85, p=0.43$, partial $\eta^{2}=0.04$; interaction: $F(2,44)=0.19, p=0.83$, partial $\eta^{2}=0.008$; Fig. 1c), movement times (cTBS: $F(2,44)=0.21, p=0.81$, partial $\eta^{2}=0.009$; interaction: $F(2,44)=0.78, p=0.46$, partial $\eta^{2}=0.03$; Fig. 1d), radial (cTBS: $F(2,44)=0.79, p=0.46$, partial $\eta^{2}=0.04$; interaction: $F(2,44)=1.08, p=0.35$, partial $\eta^{2}=0.05$; Fig. 1e) and angular error (main effect of cTBS: $F(2,44)=1.18, p=0.32$, partial $\eta^{2}=0.05$; interaction: $F(2,44)=0.16, p=0.86$, partial $\eta^{2}=0.007$; Fig. 1f). This suggests that cTBS over vmPFC or SMA had no effect on behaviour. However, since the frequentist approach has inherent limitations regarding evidence for or against null effects, we performed post-hoc Bayesian analyses on our behavioural variables, and results are reported in Table 1 for each model considered.

Comparing the candidate models using $\mathrm{BF}_{10}$, we see that the evidence in favour of the reward-only model is highest for both reaction times $\left(\mathrm{BF}_{10}=16.9\right)$ and selection accuracy $\left(\mathrm{BF}_{10}=7.76 \mathrm{e}+4\right)$, as well as peak velocity and movement time $\left(\mathrm{BF}_{10}=7.88 \mathrm{e}+7\right.$ and $\left.7.75 \mathrm{e}+5\right)$. These results are in line with the earlier frequentist analyses. In contrast, while the evidence pointed toward the null model for radial and angular error, the evidence against the reward-only model was weak, with $\mathrm{BF}_{10}=0.53$ and $\mathrm{BF}_{10}=0.63$, respectively. According to Wagenmakers et al. (2011), this represents only "anecdoctal" evidence for the null, emphasising the inconclusiveless of this result. Specifically, angular and radial accuracy were slightly lower in the reward condition compared to no reward (Fig. 1e, f). 
To assess the impact of cTBS on performance, we included $\mathrm{BF}_{10}$ for three additional candidate models: $\mathrm{y} \sim \mathrm{cTBS}, \mathrm{y} \sim \mathrm{cTBS}+$ reward, and $\mathrm{y} \sim \mathrm{cTBS} *$ reward (including an interaction). However, as a natural consequence of the strong evidence in favour of a reward effect, the $\mathrm{BF}_{10}$ of all variables tended to be very low for the cTBS-only model and extremely high for the models that included reward. To account for this, we compared Bayes factors with respect

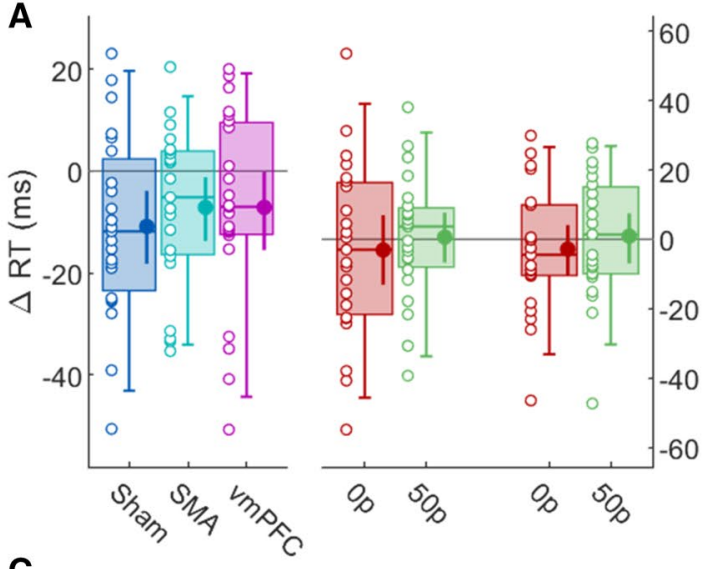

C

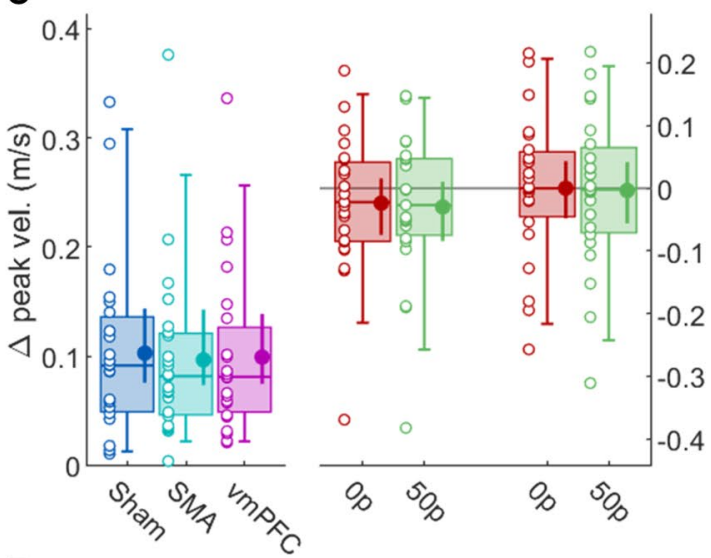

$\mathbf{E}$

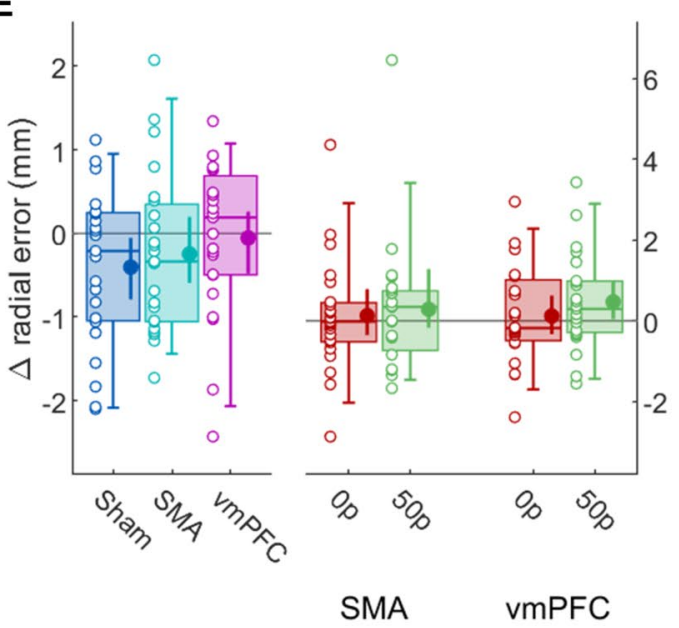

Fig. 2 Effect of reward and cTBS on different behavioural variables. a Reaction times. On the left, 50p trials performance for each cTBS group are normalised to $0 \mathrm{p}$ trials (i.e. reward-normalised), and on the right $0 \mathrm{p}$ and $50 \mathrm{p}$ trials for each cTBS group are normalised to sham performance (i.e. sham-normalised). The empty dots represent indi-
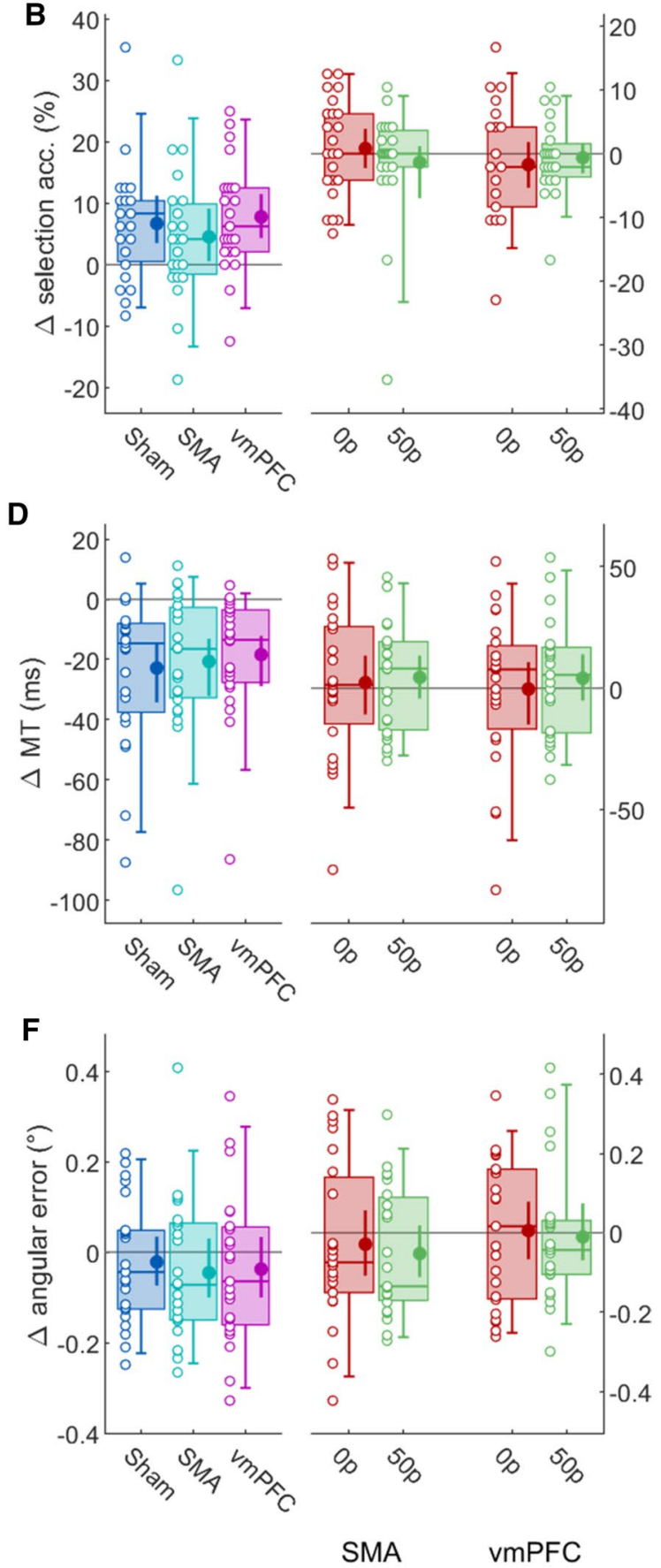

vidual values for each group and the box plots indicate the [5-25-5075-95] percentiles. The filled dot and and the error bars indicate the mean and bootstrapped $95 \%$ CIs of the mean. b-f Other variables in the same format as a 
Table 1 Bayesian model comparison for kinematics variables
Fig. 3 Model-averaged posterior $\beta$ coefficients for peak velocity. All effects are centred on 0 because they are experessed as a function of the model's intercept. Bars on top of each probability density function indicate the $90 \%$ highest density interval. a Posterior distributions for each cTBS condition, $\mathbf{b}$ for each reward condition, $\mathbf{c}$ for each possible interaction

\begin{tabular}{|c|c|c|c|c|c|c|}
\hline Model & $\mathrm{Y} \sim$ & Null (incl. pt.) & Reward & cTBS & cTBS + reward & cTBS $\times$ reward \\
\hline \multirow[t]{3}{*}{ Reaction times } & $\mathrm{p}$ (Mldata) & 0.051 & 0.871 & 0.004 & 0.064 & 0.009 \\
\hline & $\mathrm{BF}_{10}$ & 1 & 16.966 & 0.076 & 1.253 & 0.180 \\
\hline & $\mathrm{BF}_{\text {best }}$ & 0.059 & 1 & 0.004 & 0.074 & 0.011 \\
\hline \multirow[t]{3}{*}{ Selection accuracy } & p(Mldata) & $1.150 \mathrm{e}-5$ & 0.893 & $1.046 \mathrm{e}-6$ & 0.089 & 0.018 \\
\hline & $\mathrm{BF}_{10}$ & 1 & $77,668.98$ & 0.091 & 7734.165 & 1562.092 \\
\hline & $\mathrm{BF}_{\text {best }}$ & $1.288 \mathrm{e}-5$ & 1 & $1.171 \mathrm{e}-6$ & 0.1 & 0.02 \\
\hline \multirow[t]{3}{*}{ Peak velocity } & p(Mldata) & $9.977 e-9$ & 0.786 & $1.759 \mathrm{e}-9$ & 0.191 & 0.023 \\
\hline & $\mathrm{BF}_{10}$ & 1 & $7.881 \mathrm{e}+7$ & 0.176 & $1.912 \mathrm{e}+7$ & $2.299 \mathrm{e}+6$ \\
\hline & $\mathrm{BF}_{\text {best }}$ & $1.269 \mathrm{e}-8$ & 1 & $2.238 \mathrm{e}-9$ & 0.243 & 0.029 \\
\hline \multirow[t]{3}{*}{ Movement times } & $\mathrm{p}$ (Mldata) & $1.168 \mathrm{e}-6$ & 0.906 & $1.029 \mathrm{e}-7$ & 0.082 & 0.012 \\
\hline & $\mathrm{BF}_{10}$ & 1 & 775,804 & 0.088 & $70,418.68$ & 9941.661 \\
\hline & $\mathrm{BF}_{\text {best }}$ & $1.289 \mathrm{e}-6$ & 1 & $1.136 \mathrm{e}-7$ & 0.091 & 0.013 \\
\hline \multirow[t]{3}{*}{ Radial error } & p(Mldata) & 0.532 & 0.285 & 0.112 & 0.06 & 0.011 \\
\hline & $\mathrm{BF}_{10}$ & 1 & 0.535 & 0.21 & 0.113 & 0.022 \\
\hline & $\mathrm{BF}_{\text {best }}$ & 1 & 0.535 & 0.21 & 0.113 & 0.022 \\
\hline \multirow[t]{3}{*}{ Angular error } & p(Mldata) & 0.473 & 0.302 & 0.124 & 0.089 & 0.012 \\
\hline & $\mathrm{BF}_{10}$ & 1 & 0.639 & 0.262 & 0.188 & 0.024 \\
\hline & $\mathrm{BF}_{\text {best }}$ & 1 & 0.639 & 0.262 & 0.188 & 0.024 \\
\hline
\end{tabular}

All models include participants as a random variables. The propability $\mathrm{p}$ (Mldata) of a model given our dataset indicates which model is most likely compared to all other models considered. The most likely model for each variable is highlighted in bold. Bayesian factors $\left(\mathrm{BF}_{10}\right)$ are the ratio between posterior like-

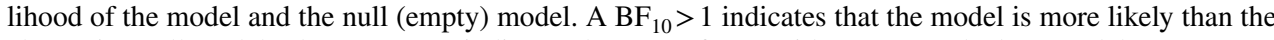
alternative null model. The $\mathrm{BF}_{\text {best }}$ row indicates the Bayes factor with respect to the best model
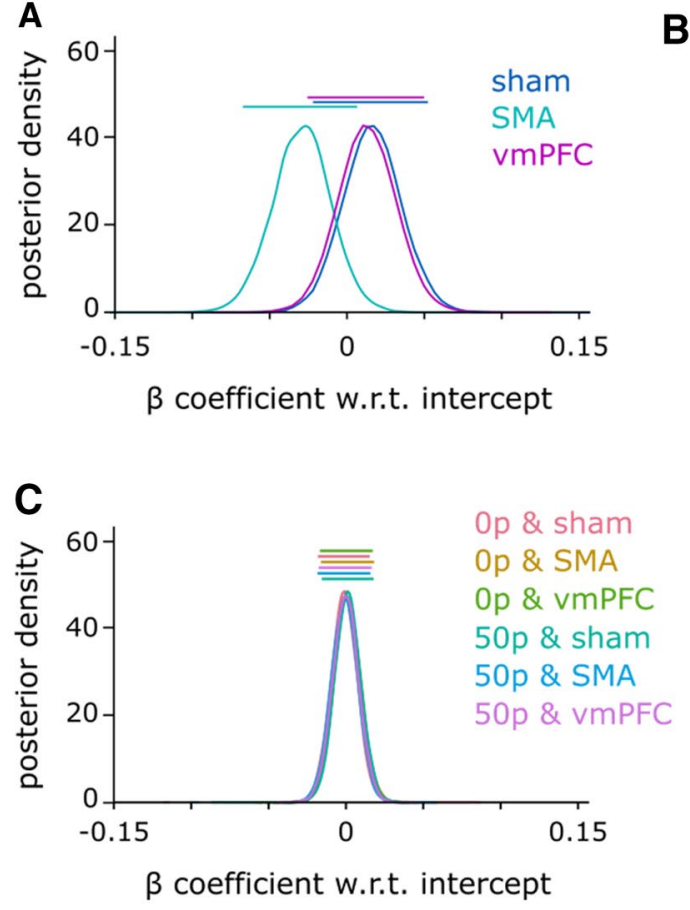

to the best model rather than the null model $\left(\mathrm{BF}_{\text {best }}\right)$, which is tantamount to assessing how close the evidence for the considered model and best model is. Note that this method is uninformative for radial and angular error, because the
B

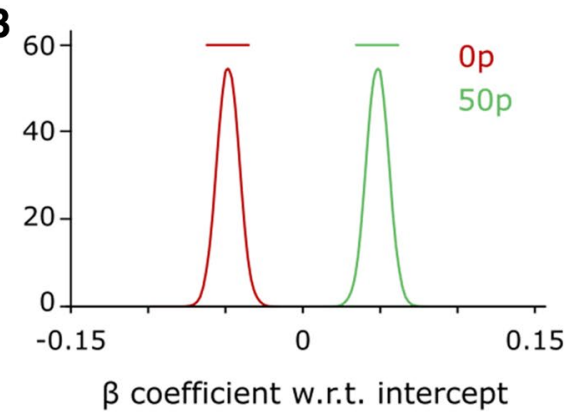

null model is already the best, and because the $\mathrm{BF}_{10}$ remains weak (i.e. anecdotal) for all models anyway (Fig. 2).

The cTBS + reward model exhibited strong evidence toward the null for all variables, except peak 
velocity, for which evidence toward the null was still strong $\left(\mathrm{BF}_{\text {best }}=0.176\right)$ but less compelling than for the other variables. To assess which cTBS condition may drive this lower $\mathrm{BF}_{\text {best }}$, we assessed the model-averaged posterior distribution of each condition's $\beta$ coefficient (Fig. 3). Posterior effect sizes with respect to cTBS (Fig. 3a) indicate that this may be due to a small deviation of the SMA group effect size compared to sham and vmPFC. In comparison, the posterior effect size for reward showed a strong contrast between 0 and 50p (Fig. 3b), as expected from the high $\mathrm{BF}_{10}$ for the reward-only model (Table 1 ). To assess whether there was an indirect impact of cTBS on reward-driven effects, we also considered the full cTBS*reward model. However, there was consistent and extreme evidence against this model compared to the best model for all variables considered (all $\mathrm{BF}_{\text {best }}<0.03$ ), excluding the possibility that cTBS manipulation had an impact in this task, directly or on the reward-driven effect. Illustrating this strong evidence against a potential interaction on peak velocity, the posterior coefficients for interactions were entirely overlapping (Fig. 3c).
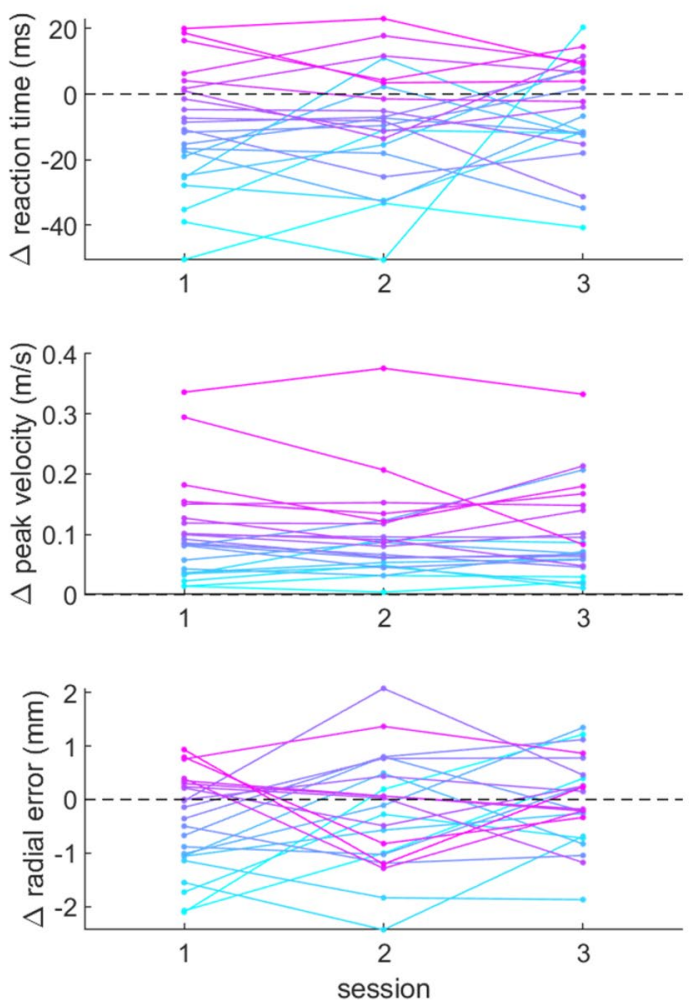

Fig. 4 Reward-driven effect on kinematic variables across multi-day sessions. Values indicate the average change with reward for each participant, with a positive value indicating an increase compared to no-reward trials. Each line represents a participant across each of the three cTBS sessions, with sessions 1-3 indicating the chronological

\section{Effect of reward over multi-day sessions}

To assess whether the reward effect on behavioural variables was reliable across multi-day sessions, we then observed how the average difference between rewarded and non-rewarded trials fluctuated over chronological sessions. Because the order of each target cTBS session was counterbalanced across participant, the same combination of cTBS session is represented in each of the chronological session. The kinematic variables we assessed can be seen in Fig. 4. We performed a repeated-measure Bayesian ANOVA, which shows substantial evidence against an effect of session on reaction times $\left(\mathrm{BF}_{10}=0.293\right)$, peak velocity $\left(\mathrm{BF}_{10}=0.139\right)$, movement time $\left(\mathrm{BF}_{10}=0.155\right)$ and angular error $\left(\mathrm{BF}_{10}=0.135\right)$, and anecdotal evidence against an effect of session as well on selection accuracy $\left(\mathrm{BF}_{10}=0.503\right)$ and radial error $\left(\mathrm{BF}_{10}=0.420\right)$. These statistical results show that there is no evidence in favour of a differential effect of reward across multi-day session, indicating that the beneficial effects of reward on motor control were reliable across multi-day sessions.
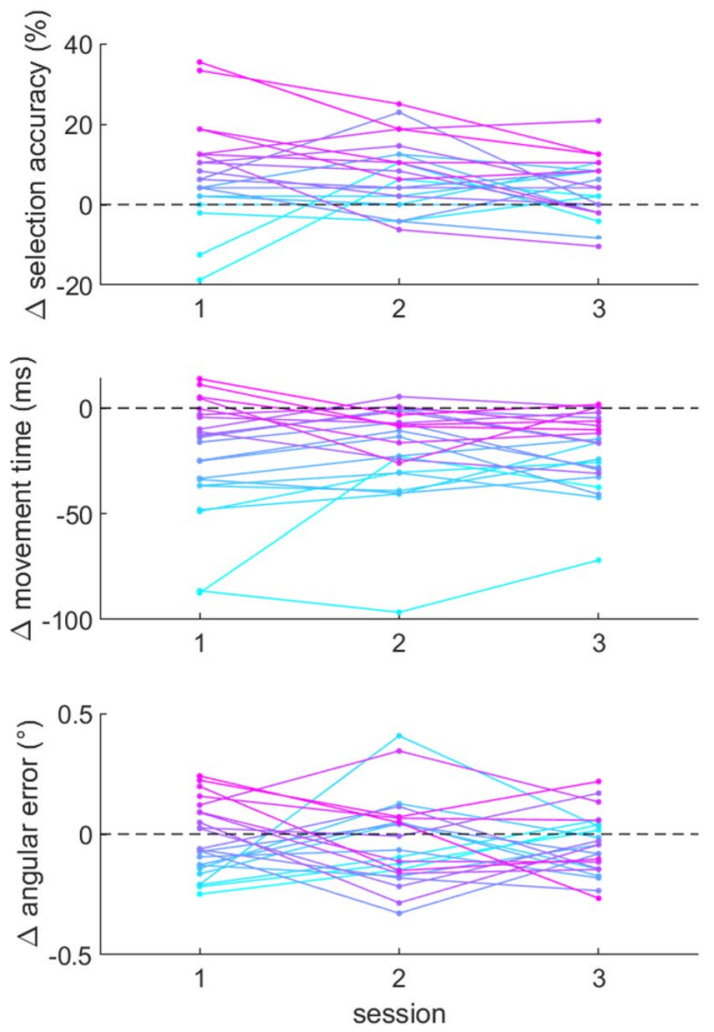

order rather than target cTBS region. As the cTBS session order was counterbalanced across participants, each chronological session contains the same combination of vmPFC, SMA and sham cTBS. There was no significant effect of session number for all of the variables displayed here 
A

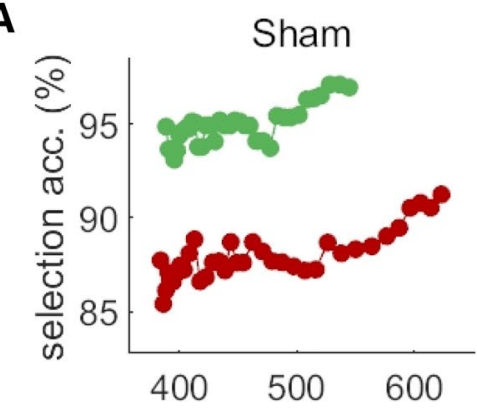

D

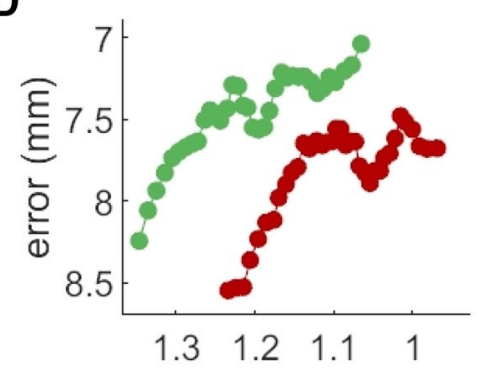

B

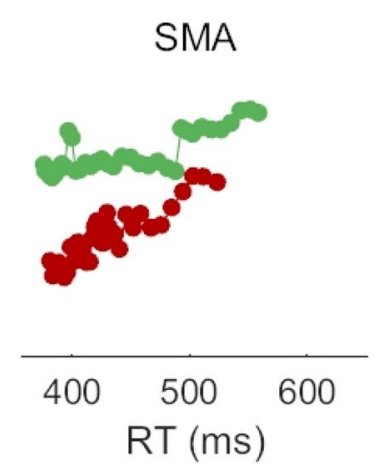

E

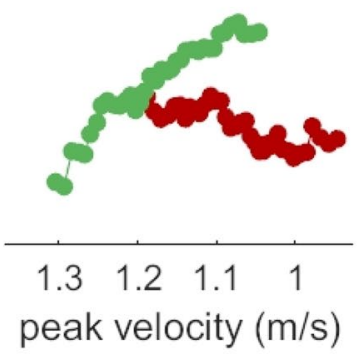

C

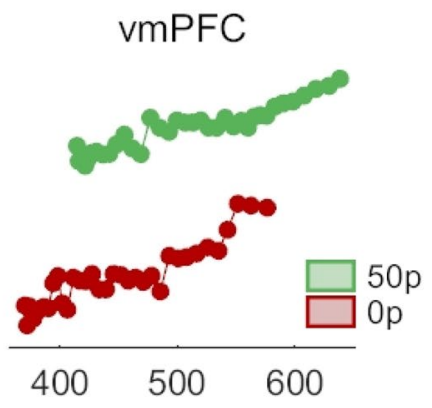

$\mathbf{F}$

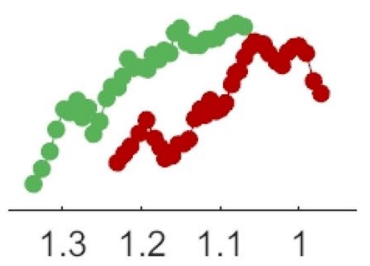

Fig. 5 Speed-accuracy functions for each reward and cTBS condition. The selection $(\mathbf{a}-\mathbf{c})$ and execution $(\mathbf{d}-\mathbf{f})$ speed-accuracy functions are the top three and bottom three panels, respectively. The functions are obtained by sliding a $30 \%$ centile-wide window over 50 quantilebased bins and averaging each bin across participant. For the selec- tion panels, the count of non-distracted trials and distracted trials for each bin was obtained, and the ratio $(100 *$ non-distracted/total $)$ calculated afterwards. Note that the axes of the execution functions are reversed so that high speed and low accuracy are on the bottom-left corner like for the selection functions
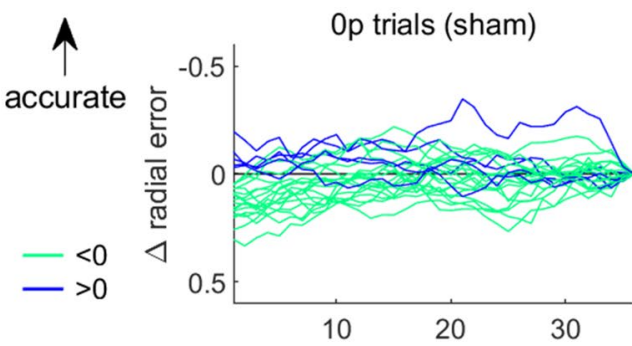

Op trials (SMA TMS)

$50 p$ trials (SMA TMS)
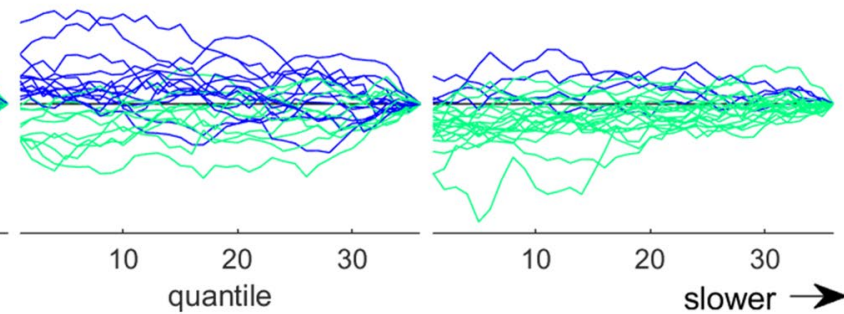

Fig. 6 Individual speed-accuracy functions for the no-reward condition of the SMA cTBS group (middle) and for two control groups (right and left). The functions are obtained by sliding a $30 \%$ centile window over 50 quantile-based bins. Each individual profile

\section{Effect of reward and cTBS on speed-accuracy functions}

Next, we assessed the speed-accuracy functions of the selection and execution components in all cTBS conditions. As can be seen in Fig. 5, we can consistently see a shift in the speed-accuracy functions of both these components with reward, in line with previous results (Fig. 5a-f). However, the execution speed-accuracy function in the SMA cTBS group does not exhibit a normal profile at baseline (0p trials; is normalised to its end value. Profiles exhibiting an increase and a decrease in accuracy with slower movements are plotted in light green and blue, respectively

Fig. 5e). Instead, radial error appears to be maintained across the range of peak velocities displayed. However, this profile did not extend to rewarded trials. Because this behaviour at baseline is surprising, we examined individual speed-accuracy profiles for this condition to ensure this was not driven by outliers. We can observe from Fig. 6 that indeed, two participants displayed more accurate performance at high speeds for 0p trials in the SMA cTBS condition (middle panel), compared to the majority of participants. However, overall, there were also more participants who exhibited 
more accurate performance at higher speeds in this condition than in comparable conditions, such as $0 p$ trials in the sham condition (Fig. 6, left panel) or the 50p trials in the SMA cTBS condition (right panel). Therefore, while no clear speed-accuracy trade-off was observed for the $0 \mathrm{p}$ trials in the SMA cTBS condition, it cannot be conclusively stated that this was driven by outliers. A possible reason for this unexpected result is that it is driven by the small, noisy trend observed for peak velocities illustrated in Fig. 3a. However, as demonstrated by the Bayes factor for peak velocity, this result remains too marginal to draw any strong conclusion.

\section{Discussion}

In this study, we employed cTBS with the aim of perturbing activity in the vmPFC and SMA in order to modulate previously characterised reward-driven effects on selection and execution performance in a reaching task. While the effects of reward characterised in Codol et al. (2020) were reliably reproduced within participants and across a series of four sessions held on different days, cTBS stimulation of either of the two target regions did not result in any alteration of these effects.

The replication of reward-driven effects on a reaching task across weekly sessions and on the same individuals confirms the conclusions from our previous study (Codol et al. 2020). While it could be argued that this is natural considering that we pre-selected participants, it was not granted that an effect found on one day for a given participant could replicate consistently in a subsequent session held on another day. Nevertheless, one divergent result is that in this study we observe a reduction in reaction times with reward, in Codol et al. (2020) no significant effect had been observed despite a larger sample size $(N=30)$. However, a similar trend that failed to reach significance had been observed. Here, pre-selecting participants may have allowed that trend to reach the significance threshold, suggesting that there is an effect of reward on reaction times, although it is likely a small effect size.

Interpreting the absence of any cTBS impact of the reward-driven effects is less straightforward, as drawing conclusions on the sole basis of non-significant results is a well-established fallacy (Altman and Bland 1995). To gain a better understanding of the data, we performed a series of a posteriori Bayesian ANOVA analyses, allowing us to determine if the non-significant results are actually null results. However, this does not negate the inconclusive nature of a null result per se. Therefore, the rest of this discussion is merely speculative rather than conclusive, although it can provide additional information to support previously reported evidence.
First, the absence of an effect of vmPFC stimulation could suggest that other regions may influence the selection component of motor control. As mentioned previously, early sensory areas such as visual cortices are possible candidates (Anderson 2016; Goard and Dan 2009; Pinto et al. 2013; Tosoni et al. 2013). However, prefrontal regions show a very complex hierarchical organisation for reward information processing (Hunt and Hayden 2017), and other possibilities should not be overlooked. It could be for instance that other well-known reward-processing centres located in the prefrontal areas are involved in processing the selection aspects of motor control, such as the cingulate cortex (Blair et al. 2013; Klein-Flugge et al. 2016; Tosoni et al. 2013), which is unfortunately not a possible target for cTBS stimulation due to its deep anatomical location. Another possibility is that vmPFC is indeed involved in the selection process, but that the processing network allows for some compensatory activity, meaning that perturbing vmPFC activity does not affect the network capacity as a whole. Finally, it could be that vmPFC is involved in selection but cTBS is not as effective in perturbing neural activity in vmPFC as in other regions. To our knowledge, only one study reports a significant effect of repetitive cTBS on vmPFC (Lev-Ran et al. 2012), suggesting that perturbation of neural activity with this technique is possible. However it cannot be ascertained whether our specific stimulation protocol can do so successfully in a sustained fashion across the duration of the behavioural task $(20 \mathrm{~min})$. While the study from LevRanand et al. (2012) stimulated participants every $15 \mathrm{~min}$, the experiment presented here lasted about $15 \mathrm{~min}$ as well, suggesting that an effect would have sustained for sufficient time after stimulation ceased. Overall, it is not clear based on our results whether the observed inhibitory effects triggered by M1 cTBS (Huang et al. 2005) and SMA cTBS (Legon et al. 2013) can generalise to vmPFC stimulation (Castrillon et al. 2020). Additionally, a large study (56 participants) showed that even though cTBS can have an effect on M1, this effect is highly variable across participants and depends on a variety of individual factors (Hamada et al. 2013), potentially explaining the lack of an observed effect here. It is also unclear whether the heterogeneity of individual responses to cTBS of M1 is mirrored when targeting other brain regions, such as those employed here.

The situation is less ambiguous regarding the absence of an effect of cTBS stimulation on SMA. First, there are numerous studies showing cTBS influences SMA activity (Arai et al. 2011; Legon et al. 2013; Matsunaga et al. 2005; Shirota et al. 2012; Zenon et al. 2015), some of them showing that stimulation can also modulate downstream regions such as M1 (Arai et al. 2011, 2012; Matsunaga et al. 2005; Shirota et al. 2012). This last point indicates that any cTBS effect should be strong enough to lead to consequences even in regions that were not directly stimulated. Additionally, 
the non-conclusive trend we observe in the peak velocity posteriors with SMA stimulation (Fig. 3a), and the altered speed-accuracy function (Fig. 5e) are both in line with the possibility of a global cTBS effect on action vigour-though a larger sample size may be required to reliably expose it. However, due to the "drawer effect" bias (Open Science Collaboration 2015), it is difficult to ascertain to which extent cTBS stimulation can reproducibly perturb neural processing of SMA. Nevertheless, considering the large set of available studies showing a significant effect of cTBS, and the inconclusive results we report of cTBS on peak velocity and speed-accuracy functions, it is more plausible that other regions implement reward-driven effects on execution, rather than to assume that cTBS is ineffective in manipulating SMA activity. Mainly, the pre-motor area and M1 represent potential alternative candidates. The premotor area is central to movement planning and several studies have shown its sensitivity to reward (Ramkumar et al. 2016; Roesch and Olson 2003, 2004). Regarding M1, a large literature demonstrates effects of reward on various aspects of M1 processing (Bundt et al. 2016; Galaro et al. 2019; Kapogiannis et al. 2008; Mawase et al. 2016, 2017; Ramkumar et al. 2016; Thabit et al. 2011), making it a suitable candidate for mediating the reward-driven effects observed in our study. Furthermore, we show in Codol et al. (2020) that some execution improvements may be due to an increase in feedback control, likely transcortical (Omrani et al. 2016; Pruszynski et al. 2011) and visuomotor feedback (Carroll et al. 2019). Interestingly, transcortical feedback relies on M1 modulation (Pruszynski et al. 2011), in line with the possibility that M1 supports reward-driven improvements in execution.

Overall, this study shows that the reward-driven effects on reaching are robust and replicable across multiple sessions for a given participant. However, cTBS on the vmPFC and SMA was ineffective in manipulating these effects. While it is difficult to interpret this absence of cTBS effects, we outline possible explanations for this. Notably, the absence of effect following SMA cTBS further bolsters the possibility that reward impacts motor execution at a late stage of the sensorimotor loop, likely at the level of the premotor area or M1.

Acknowledgements This work was supported by the European Research Council Grant MotMotLearn 637488.

Author contributions $\mathrm{OC}, \mathrm{JMG}$ and $\mathrm{PJH}$ conceived and designed the research, $\mathrm{OC}$ and $\mathrm{RJ}$ acquired the data, RJ analysed the imaging data, OC analysed the behavioural data, OC, JMG and PJH interpreted the results, OC wrote the first draft, OC, JMG and PJH edited the manuscript, OC, JMG, RJ and PJH approved the final version of the manuscript.

Open Access This article is licensed under a Creative Commons Attribution 4.0 International License, which permits use, sharing, adaptation, distribution and reproduction in any medium or format, as long as you give appropriate credit to the original author(s) and the source, provide a link to the Creative Commons licence, and indicate if changes were made. The images or other third party material in this article are included in the article's Creative Commons licence, unless indicated otherwise in a credit line to the material. If material is not included in the article's Creative Commons licence and your intended use is not permitted by statutory regulation or exceeds the permitted use, you will need to obtain permission directly from the copyright holder. To view a copy of this licence, visit http://creativecommons.org/licenses/by/4.0/.

\section{References}

Altman DG, Bland JM (1995) Absence of evidence is not evidence of absence. BMJ 311:485

Anderson BA (2016) The attention habit: how reward learning shapes attentional selection: the attention habit. Ann N Y Acad Sci 1369:24-39

Arai N, Muller-Dahlhaus F, Murakami T, Bliem B, Lu M-K, Ugawa Y, Ziemann U (2011) State-dependent and timing-dependent bidirectional associative plasticity in the human SMA-M1 network. J Neurosci 31:15376-15383

Arai N, Lu M-K, Ugawa Y, Ziemann U (2012) Effective connectivity between human supplementary motor area and primary motor cortex: a paired-coil TMS study. Exp Brain Res 220:79-87

Arnsten AFT (1998) Catecholamine modulation of prefrontal cortical cognitive function. Trends Cognit Sci 2:436-447

Berret B, Castanier C, Bastide S, Deroche T (2018) Vigour of selfpaced reaching movement: cost of time and individual traits. Sci Rep 8:10655

Blair KS, Otero M, Teng C, Jacobs M, Odenheimer S, Pine DS, Blair RJR (2013) Dissociable roles of ventromedial prefrontal cortex (vmPFC) and rostral anterior cingulate cortex (rACC) in value representation and optimistic bias. NeuroImage 78:103-110

Bundt C, Abrahamse EL, Braem S, Brass M, Notebaert W (2016) Reward anticipation modulates primary motor cortex excitability during task preparation. NeuroImage 142:483-488

Carroll TJ, McNamee D, Ingram JN, Wolpert DM (2019) Rapid visuomotor responses reflect value-based decisions. J Neurosci 39:3906-3920

Castiello U (2005) The neuroscience of grasping. Nat Rev Neurosci 6:726-736

Castrillon G, Sollmann N, Kurcyus K, Razi A, Krieg SM, Riedl V (2020) The physiological effects of noninvasive brain stimulation fundamentally differ across the human cortex. Sci Adv 6:eaay 2739

Codol O, Holland PJ, Manohar SG, Galea JM (2020) Reward-based improvements in motor control are driven by multiple errorreducing mechanisms. J Neurosci. https://doi.org/10.1523/ JNEUROSCI.2646-19.2020

Daw ND, Niv Y, Dayan P (2005) Uncertainty-based competition between prefrontal and dorsolateral striatal systems for behavioral control. Nat Neurosci 8:1704-1711

Daw ND, O'Doherty JP, Dayan P, Seymour B, Dolan RJ (2006) Cortical substrates for exploratory decisions in humans. Nature 441:876-879

Galaro JK, Celnik P, Chib VS (2019) Motor cortex excitability reflects the subjective value of reward and mediates its effects on incentive-motivated performance. J Neurosci 39:1236-1248

Galea JM, Albert NB, Ditye T, Miall RC (2010) Disruption of the dorsolateral prefrontal cortex facilitates the consolidation of procedural skills. J Cognit Neurosci 22:1158-1164

Goard M, Dan Y (2009) Basal forebrain activation enhances cortical coding of natural scenes. Nat Neurosci 12:1444-1449 
Graybiel AM (2008) Habits, rituals, and the evaluative brain. Annu Rev Neurosci 31:359-387

Griffiths B, Beierholm UR (2017) Opposing effects of reward and punishment on human vigor. Sci Rep 7:42287

Hamada M, Murase N, Hasan A, Balaratnam M, Rothwell JC (2013) The role of interneuron networks in driving human motor cortical plasticity. Cereb Cortex 23:1593-1605

Hendrix CM, Campbell BA, Tittle BJ, Johnson LA, Baker KB, Johnson MD, Molnar GF, Vitek JL (2018) Predictive encoding of motor behavior in the supplementary motor area is disrupted in parkinsonism. J Neurophysiol 120:1247-1255

Hikosaka O, Nakamura K, Sakai K, Nakahara H (2002) Central mechanisms of motor skill learning. Curr Opin Neurobiol $12: 217-222$

Huang Y-Z, Edwards MJ, Rounis E, Bhatia KP, Rothwell JC (2005) Theta burst stimulation of the human motor cortex. Neuron 45:201-206

Hunt LT, Hayden BY (2017) A distributed, hierarchical and recurrent framework for reward-based choice. Nat Rev Neurosci 18:172-182

Jenkinson M, Smith S (2001) A global optimisation method for robust affine registration of brain images. Med Image Anal 5:143-156

Jenkinson M, Bannister P, Brady M, Smith S (2002) Improved optimization for the robust and accurate linear registration and motion correction of brain images. NeuroImage 17:825-841

Kapogiannis D, Campion P, Grafman J, Wassermann EM (2008) Reward-related activity in the human motor cortex. Eur J Neurosci 27:1836-1842

Klein-Flugge MC, Kennerley SW, Friston K, Bestmann S (2016) Neural signatures of value comparison in human cingulate cortex during decisions requiring an effort-reward trade-off. J Neurosci 36:10002-10015

Kojima Y, Soetedjo R (2017) Selective reward affects the rate of saccade adaptation. Neuroscience 355:113-125

Legon W, Dionne JK, Staines WR (2013) Continuous theta burst stimulation of the supplementary motor area: effect upon perception and somatosensory and motor evoked potentials. Brain Stimul 6:877-883

Lev-Ran S, Shamay-Tsoory SG, Zangen A, Levkovitz Y (2012) Transcranial magnetic stimulation of the ventromedial prefrontal cortex impairs theory of mind learning. Eur Psychiatry 27:285-289

Manohar SG, Chong TT-J, Apps MAJ, Batla A, Stamelou M, Jarman PR, Bhatia KP, Husain M (2015) Reward pays the cost of noise reduction in motor and cognitive control. Curr Biol 25:1707-1716

Manohar SG, Muhammed K, Fallon SJ, Husain M (2019) Motivation dynamically increases noise resistance by internal feedback during movement. Neuropsychologia 123:19-29

Matsunaga K, Maruyama A, Fujiwara T, Nakanishi R, Tsuji S, Rothwell JC (2005) Increased corticospinal excitability after 5 $\mathrm{Hz}$ rTMS over the human supplementary motor area. J Physiol 562:295-306

Mawase F, Wymbs N, Uehara S, Celnik P (2016) Reward gain model describes cortical use-dependent plasticity. In: 2016 38th annual international conference of the IEEE engineering in medicine and biology society (EMBC), Orlando, FL, USA: IEEE, pp 5-8

Mawase F, Uehara S, Bastian AJ, Celnik P (2017) Motor learning enhances use-dependent plasticity. J Neurosci 37:2673-2685

Mazzoni P, Hristova A, Krakauer JW (2007) Why don't we move faster? Parkinson's disease, movement vigor, and implicit motivation. J Neurosci 27:7105-7116

Omrani M, Murnaghan CD, Pruszynski JA, Scott SH (2016) Distributed task-specific processing of somatosensory feedback for voluntary motor control. ELife 5

Open Science Collaboration (2015) Estimating the reproducibility of psychological science. Science 349:aac4716-aac4716

Pinto L, Goard MJ, Estandian D, Xu M, Kwan AC, Lee S-H, Harrison TC, Feng G, Dan Y (2013) Fast modulation of visual perception by basal forebrain cholinergic neurons. Nat Neurosci 16:1857-1863

Pruszynski JA, Kurtzer I, Nashed JY, Omrani M, Brouwer B, Scott SH (2011) Primary motor cortex underlies multi-joint integration for fast feedback control. Nature 478:387-390

Ramkumar P, Dekleva B, Cooler S, Miller L, Kording K (2016) Premotor and motor cortices encode reward. PLoS ONE 11:e0160851

Rascol O, Sabatini U, Chollet F, Fabre N, Senard JM, Montastruc JL, Celsis P, Marc-Vergnes JP, Rascol A (1994) Normal activation of the supplementary motor area in patients with Parkinson's disease undergoing long-term treatment with levodopa. J Neurol Neurosurg Psychiatry 57:567-571

Reppert TR, Rigas I, Herzfeld DJ, Sedaghat-Nejad E, Komogortsev O, Shadmehr R (2018) Movement vigor as a traitlike attribute of individuality. J Neurophysiol 120:741-757

Roesch MR, Olson CR (2003) Impact of expected reward on neuronal activity in prefrontal cortex, frontal and supplementary eye fields and premotor cortex. J Neurophysiol 90:1766-1789

Roesch MR, Olson CR (2004) Neuronal activity related to reward value and motivation in primate frontal cortex. Science 304:307-310

Rorden C, Brett M (2000) Stereotaxic display of brain lesions. Behav Neurol 12:191-200

Shadmehr R, Krakauer JW (2008) A computational neuroanatomy for motor control. Exp Brain Res 185:359-381

Shirota Y, Hamada M, Terao Y, Ohminami S, Tsutsumi R, Ugawa Y, Hanajima R (2012) Increased primary motor cortical excitability by a single-pulse transcranial magnetic stimulation over the supplementary motor area. Exp Brain Res 219:339-349

Sohn J, Lee D (2006) Effects of reward expectancy on sequential eye movements in monkeys. Neural Netw 19:1181-1191

Stanford AD, Luber B, Unger L, Cycowicz YM, Malaspina D, Lisanby SH (2013) Single pulse TMS differentially modulates reward behavior. Neuropsychologia 51:3041-3047

Summerside EM, Shadmehr R, Ahmed AA (2018) Vigor of reaching movements: reward discounts the cost of effort. J Neurophysiol 119:2347-2357

Takikawa Y, Kawagoe R, Itoh H, Nakahara H, Hikosaka O (2002) Modulation of saccadic eye movements by predicted reward outcome. Exp Brain Res 142:284-291

Thabit MN, Nakatsuka M, Koganemaru S, Fawi G, Fukuyama H, Mima $\mathrm{T}$ (2011) Momentary reward induce changes in excitability of primary motor cortex. Clin Neurophysiol 122:1764-1770

Thorpe SJ, Fabre-Thorpe M (2001) Seeking categories in the brain. Science 291:260-263

Tosoni A, Shulman GL, Pope ALW, McAvoy MP, Corbetta M (2013) Distinct representations for shifts of spatial attention and changes of reward contingencies in the human brain. Cortex 49:1733-1749

Wagenmakers E-J, Wetzels R, Borsboom D, van der Maas HLJ (2011) Why psychologists must change the way they analyze their data: the case of psi: comment on Bem (2011). J Pers Soc Psychol 100:426-432

Zenon A, Sidibe M, Olivier E (2015) Disrupting the supplementary motor area makes physical effort appear less effortful. J Neurosci 35:8737-8744

Zhao Y, Hessburg JP, Asok Kumar JN, Francis JT (2018) Paradigm shift in sensorimotor control research and brain machine interface control: the influence of context on sensorimotor representations. Front Neurosci 12

Publisher's Note Springer Nature remains neutral with regard to jurisdictional claims in published maps and institutional affiliations. 